\title{
Electron Irradiation-Induced Defects and Phase Transformations in Two- Dimensional Transition Metal Dichalcogenides
}

\author{
Arkady V. Krasheninnikov ${ }^{1,2,3,4}$ \\ 1. Helmholtz Zentrum Dresden-Rossendorf, Institute of Ion Beam Physics and Materials Research, \\ Germany. \\ 2. Department of Applied Physics, Aalto University School of Science, Finland. \\ 3. DTU Nanotech, Technical University of Denmark, Lyngby, Denmark. \\ 4. National University of Science and Technology MISiS, Moscow, Russia.
}

Following isolation of a single sheet of graphene, many other two-dimensional (2D) systems such as hexagonal BN, transition metal dichalcogenides (TMDs) and silica bilayers were manufactured. All these systems contain defects and impurities, which may govern the electronic and optical properties of these materials. Moreover, defects can appear during the characterization of the materials in transmission electron microscope. All of these call upon the studies on defect properties and mechanisms of their formation under electron beam. In my talk, I will present the results [1-8] of our first-principles theoretical studies of defects (native and irradiation-induced) in inorganic 2D systems with main focus on transition metal dichalcogenides obtained in close collaboration with several experimental transmission electron microscopy groups.

I will also dwell upon the main mechanisms of damage production, including knock-on damage, electronic excitations, etching and their combination. I will show that electronic excitations can lower the threshold for knock-on damage, thus giving rise to production of defects at rather low electron energies when direct knock-on damage should not be possible.

I will further discuss defect- and impurity-mediated engineering of the electronic structure of inorganic 2D materials. Specifically, I will address agglomeration of vacancies into line defects [1, 3-5] and discuss how such structures influence the electronic properties of 2D inorganic materials.

Finally, I will also present the results of our theoretical studies of electron-beam induced phase transformations in 2D TMDs [7] when electric charge, mechanical strain and vacancies are present. Based on the results of our calculations, we propose an explanation for this phenomenon which is likely promoted by charge redistribution in the monolayer combined with vacancy formation due to electron beam and associated mechanical strain in the sample.

References:

[1] H.-P. Komsa and A. V. Krasheninnikov, Advanced Electronic Materials 3 (2017), p. 1600468.

[2] Y.-C. Lin et al, Adv. Func. Mater. 28 (2018), p. 1704210.

[3] X. Zhao et al, MRS Bulletin 42 (2017), p. 667.

[4] Y.-C. Lin et al, Nature Communications 6 (2015), p. 6736.

[5] O. Lehtinen et al, ACS Nano 9 (2015), p. 3274.

[6] E. Sutter et al, Nano Letters 16 (2016), p. 4410.

[7] S. Kretschmer et al, J. Phys. Chem. Lett., 8 (2017), p. 3061.

[8] T. Björkman et al, ACS Nano 10 (2016), p. 10929. 

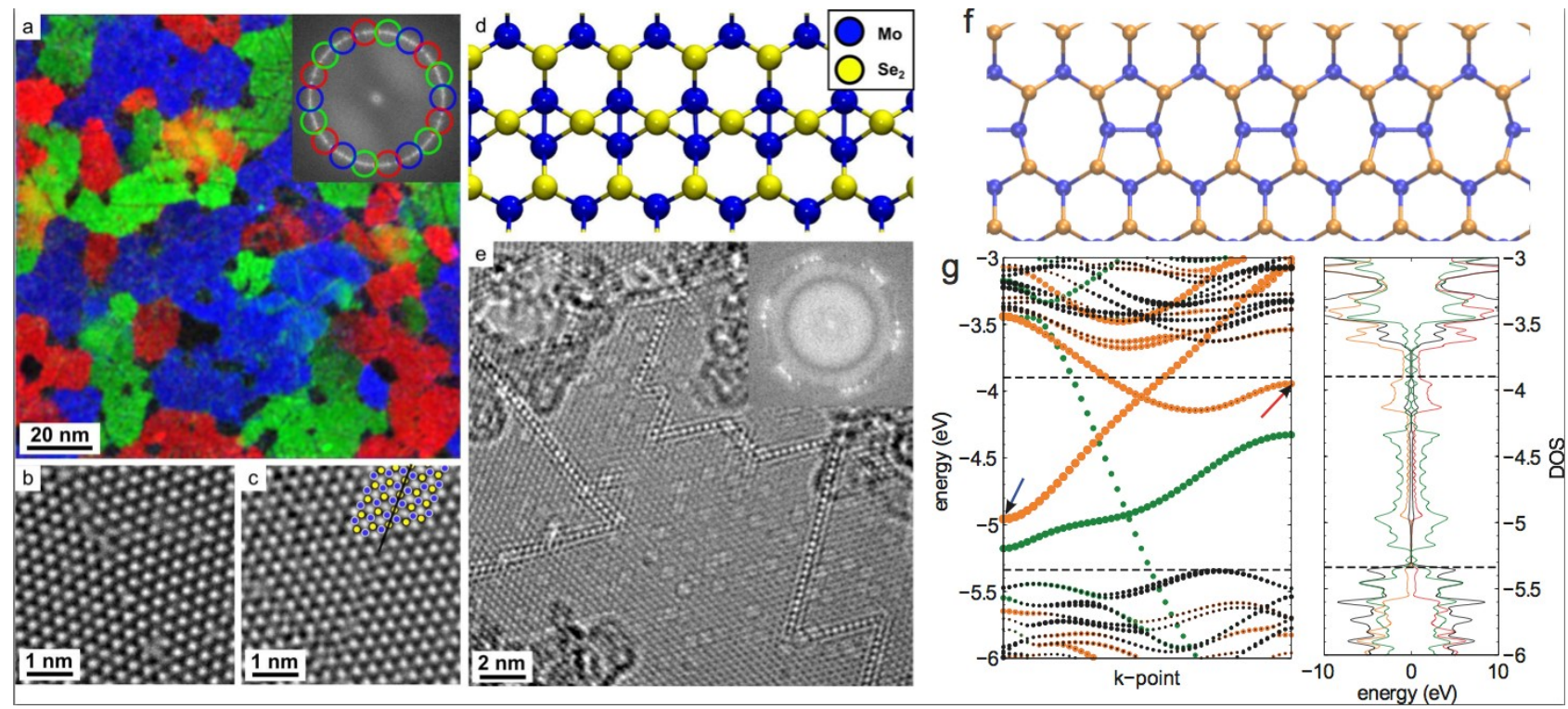

Figure 1. Line defects in inorganic two-dimensional transition metal dichalcogenides. (a) The grain structure, with the color coding corresponding to different grain orientations in MoSe2 [from Ref. 5]. (b) A close-up view of a tilt grain boundary. c: A close-view of a mirror-twin-boundary within a grain. (d) The theoretical structure, as calculated using density-functional thory. (e) A larger field of view image. Single vacancies are also visible. (f) Atomic structure of another type of mirror twin boundary structure (composed from 5-8 membered rings) observed in WSe2 [from Ref. 4]. (g) Band structure and density of states of a mirror twin boundary in W- containing transition metal dichalcogenides. 